\title{
Approximation of signals (functions) belonging to certain Lipschitz classes by almost Riesz means of its Fourier series
}

\author{
Deepmala $^{1 *}$ and Laurian-loan Piscoran ${ }^{2}$
}

"Correspondence:

dmrai23@gmail.com

'SQC and OR Unit, Indian Statistical Institute, 203 B.T. Road,

Kolkata-700 108, West Bengal, India

Full list of author information is

available at the end of the article

\section{然 Springer}

\begin{abstract}
To start with, signals are dealt with as functions of one variable and images are shown by elements of two variables. The investigation of these ideas is directly related to the transpiring area of information technology. The approximation properties of the periodic signals in $L^{r}(r \geq 1)$-spaces, Lipschitz classes $\operatorname{Lip} \alpha, \operatorname{Lip}(\alpha, r), \operatorname{Lip}(\xi(t), r)$, and a weighted Lipschitz class $W\left(L^{r}, \xi(t)\right)$ through a Fourier series, known as the Fourier approximation in the approximation theory, have wide applications in digital filters and signal analysis. The goal of our paper is to concentrate on the approximation properties of the periodic signals (functions) in the Lipschitz classes by almost Riesz means of the Fourier series associated with the function $f$. We additionally take note of the fact that our outcomes give sharper estimates than the estimates in some of the known results.
\end{abstract}

Keywords: degree of approximation; trigonometric Fourier approximation; $W\left(L^{r}, \xi(t)\right)$-class of functions; almost Riesz means

\section{Introduction}

The idea of almost convergence was presented by Lorentz in 1948 [1] and has various applications. Several authors, including Ahmad and Mursaleen [2], Lorentz [1], King [3], Mohapatra $[4,5]$, Nanda [6], and other authors [7-10] have concentrated on almost convergent sequences. As convergence motivates us to study absolute and strong convergence, similarly it was very common to study the ideas of absolute almost and strong almost convergence after the concept of almost convergence. These two concepts of absolute almost and strongly almost convergent sequences have been examined in [11] and [12], respectively.

Fourier series and operators can be considered as representations of a function or signal; they are of incredible significance in both hypothetical and practical areas. For real or complex sequences working with infinite matrices the summability methods of are of three sorts (see Maddox [13], p.185) - strong, absolute, and ordinary. Along a similar line, the idea of almost convergence must give rise to three types of summability methods strongly almost, absolutely almost and almost. The almost summable sequences have been examined in $[3,14]$ and some other references.

The error (degree) of approximation of signals $f$ belonging to the $\operatorname{Lip} \alpha, \operatorname{Lip}(\alpha, r)$, $\operatorname{Lip}(\xi(t), r)$, and $W\left(L^{r}, \xi(t)\right)$-classes by Nörlund $\left(N_{p}\right)$ and matrix summability methods has

(c) 2016 Deepmala and Piscoran. This article is distributed under the terms of the Creative Commons Attribution 4.0 International License (http://creativecommons.org/licenses/by/4.0/), which permits unrestricted use, distribution, and reproduction in any medium, provided you give appropriate credit to the original author(s) and the source, provide a link to the Creative Commons license, and indicate if changes were made. 
been addressed by different researchers, for example, Khan [15, 16], Mishra and Mishra [17], Mishra et al. [18-22], Deepmala et al. [23]. Be that as it may, till now, nothing appears to have been done as such far to get the error in approximation of signals belonging to $W\left(L^{r}, \xi(t)\right)(r \geq 1)$ space using an almost Riesz means of its Fourier series. In this manner in this paper, other results on the degree of approximation of signal $f \in W\left(L^{r}, \xi(t)\right)$ $(r \geq 1)$ by almost Riesz means of its infinite Fourier series have been built up.

Let the spaces of all convergent and bounded sequences be represented by $c$ and $l_{\infty}$, respectively. Also, $c \subset l_{\infty}$. The idea of a Banach limit comes from the well-known HahnBanach extension theorem in the theory of sequences spaces. The concept of almost convergence was introduced by Lorentz [1] as another kind of convergence by using the concept of a Banach limit.

A bounded sequence $x=\left(x_{k}\right)$ is called almost convergent to the number $\ell$ if and only if

$$
\lim _{p \rightarrow \infty}\left|\frac{1}{p} \sum_{k=n}^{n+p-1} x_{k}-l\right|=0, \quad \text { uniformly in } n .
$$

We shall represent by $F$ the space of almost convergent sequences and we write $\ell=$ $F-\lim x$.

Note that a convergent sequence is almost convergent and its limit and its generalized limit both are identical, but an almost convergent sequence need not be convergent.

For example, the sequence $z=\left(z_{n}\right)$ with

$$
z_{n}= \begin{cases}1 & \text { if } n \text { is odd } \\ 0 & \text { if } n \text { is even }\end{cases}
$$

is almost convergent to the finite limit $1 / 2$ but not convergent.

If $n=1$ in (1) then we get Cesàro summability of order 1 i.e. $(C, 1)$ convergence, and in this case we write symbolically $x_{k} \rightarrow \ell(C, 1)$; where $\ell=(C, 1)$-lim $x$.

Most of the commonly used matrix methods contain the method $F$, for example every almost convergent sequence is also a Cesàro means of order $\alpha$ i.e. $(C, \alpha)$ and the Euler means of order $\alpha$ i.e. $(E, \alpha)$-summable $(\alpha>0)$ to its $F$-limit.

Let $C[a, b]$ denote the spaces of all continuous functions $f$ defined on $[a, b]$. We know that $C[a, b]$ is a Banach space under the norm defined by $\|f\|_{\infty}:=\sup _{a \leq x \leq b}|f(x)|, f \in$ $C[a, b]$. Consider a sequence of linear operators $T_{n}: C[a, b] \rightarrow C[a, b]$. We write $T_{n}(f, x)$ for $T_{n}(f(t), x)$ and we say that its limit operator i.e. $T$ is a non-negative operator if $T(f, x) \geq$ 0 for all $f(x) \geq 0$.

For a given $2 \pi$-periodic signal $f \in L^{p}:=L^{p}[0,2 \pi], p \geq 1$, which is Lebesgue integrable. Then the Fourier series of $f(x)$ at any point ' $x$ ' given by

$$
f(x) \sim \frac{a_{0}}{2}+\sum_{n=1}^{\infty}\left(a_{n} \cos n x+b_{n} \sin n x\right) \equiv \sum_{n=0}^{\infty} A_{n}(x),
$$

with the $(n+1)$ th partial sum $s_{n}(f ; x)$ called a trigonometric polynomial of order (or degree) $n$ and given by

$$
s_{n}(f ; x):=\frac{a_{0}}{2}+\sum_{k=1}^{n}\left(a_{k} \cos k x+b_{k} \sin k x\right), \quad n \in \mathbb{N} \text { with } s_{0}(f ; x)=\frac{a_{0}}{2} .
$$


A signal $f \in \operatorname{Lip} \alpha$, if

$$
f(x+t)-f(x)=\mathrm{O}\left(\left|t^{\alpha}\right|\right) \quad \text { for } 0<\alpha \leq 1, t>0
$$

A signal $f \in \operatorname{Lip}(\alpha, r)$ for $a \leq x \leq b$ if

$$
w_{r}(t ; f)=\left\{\int_{a}^{b}|f(x+t)-f(x)|^{r} d x\right\}^{1 / r} \leq M\left(|t|^{\alpha}\right), \quad r \geq 1,0<\alpha \leq 1,
$$

where $M$ is constant (absolute positive), not essentially the same at each occurrence [24]. We have

$$
f \in \operatorname{Lip}(\xi(t), r), \quad \text { if }\left(\int_{0}^{2 \pi}|f(x+t)-f(x)|^{r} d x\right)^{1 / r}=\mathrm{O}(\xi(t)), r \geq 1, t>0 .
$$

A signal $f \in W\left(L^{r}, \xi(t)\right)[15]$ if

$$
\begin{aligned}
\omega_{r}(t ; f) & =\left(\int_{0}^{2 \pi}|f(x+t)-f(x)|^{r} \sin ^{\beta r}(x / 2) d x\right)^{1 / r} \\
& =\mathrm{O}(\xi(t)), \quad \beta \geq 0, r \geq 1, t>0
\end{aligned}
$$

where $\xi(t)$ is positive increasing function of $t$.

If $\beta=0$ then the generalized weighted Lipschitz $W\left(L^{r}, \xi(t)\right)(r \geq 1)$-class reduces to the $\operatorname{Lip}(\xi(t), r)$ class, if $\xi(t)=t^{\alpha}$ then $\operatorname{Lip}(\xi(t), r)$ class coincides with the previous class $\operatorname{Lip}(\alpha, r)$ and if $r \rightarrow \infty$ then $\operatorname{Lip}(\alpha, r)$ is converted to the $\operatorname{Lip} \alpha$ class.

The $L_{\infty}$-norm of a signal (function) $f: R \rightarrow R$ is defined by $\|f\|_{\infty}=\sup _{x \in R}\{|f(x)|\}$.

The $L_{r}$-norm of a signal (function) is defined by $\|f\|_{r}=\left(\int_{0}^{2 \pi}|f(x)|^{r} d x\right)^{1 / r}, 1 \leq r<\infty$.

The space $L_{r}[0,2 \pi]$ with $r=\infty$ includes the space $C_{2 \pi}$ of all $2 \pi$-periodic continuous functions defined on $[0,2 \pi]$, where $C_{2 \pi}$ represents the Banach space of all $2 \pi$-periodic continuous functions over $[0,2 \pi]$.

The error (degree) of approximation of a function $f: R \rightarrow R$ by a trigonometric polynomial $t_{n}$ of order $n$ under sup norm \|\|$_{\infty}$ is given by [25]

$$
\left\|t_{n}-f\right\|_{\infty}=\sup _{x \in R}\left\{\left|t_{n}(x)-f(x)\right|\right\}
$$

and $E_{n}(f)$ of a signal $f \in L_{r}$ is defined by $E_{n}(f)=\min _{n}\left\|t_{n}-f\right\|_{r}$.

This method of approximation is called a trigonometric Fourier approximation (tfa).

Let $\sum a_{n}$ be an infinite series with the sequence of its partial sums $\left\{s_{n}\right\}$. Let $\left\{p_{n}\right\}$ be a non-negative, non-decreasing, generating sequence of constants, which may be real or complex for the $\left(R, p_{n}\right)$ or $R_{p}$-method such that $P_{n}=\sum_{\gamma=0}^{n} p_{\gamma} \neq 0 \forall n \geq 0, P_{-1}=p_{-1}=0$ and $P_{n} \rightarrow \infty$ as $n \rightarrow \infty$.

The transformation (from sequence to sequence) $t_{n}=\sum_{k=0}^{n}\left(p_{k} / P_{n}\right) s_{k}$ defines the sequence $\left\{t_{n}\right\}$ of the Riesz $\left(R, p_{n}\right)$ transforms of the sequence $\left\{s_{n}\right\}$, generated by the sequence of coefficients $\left\{p_{n}\right\}$. The infinite series $\sum a_{n}$ is called Riesz summable $\left(R, p_{n}\right)$ to the sum ' $s$ ' if $\lim _{n \rightarrow \infty} t_{n}$ exists and equals a finite number ' $s$ '. 
A bounded sequence $\left\{s_{k}\right\}$ of $k$ th partial sums of an infinite Fourier series (2) is called [1] almost convergent to $s$, if

$$
\lim _{n \rightarrow \infty} s_{n, r}=\lim _{n \rightarrow \infty} \frac{s_{r}+s_{r+1}+\cdots+s_{r+n}}{n+1}=\lim _{n \rightarrow \infty} \frac{1}{(n+1)} \sum_{k=r}^{n+r} s_{k}=s,
$$

uniformly with respect to $r$.

An infinite series $\sum u_{n}$ with the sequence of its partial sums $\left\{s_{n}\right\}$ of Fourier series (2) is called almost Riesz summable [26] to a finite number ' $s$ ', provided

$$
\tau_{n, r}=\frac{1}{P_{n}} \sum_{k=0}^{n} p_{k} s_{k, r} \rightarrow s \quad \text { as } n \rightarrow \infty
$$

uniformly with respect to $r$, where $s_{k, r}=\frac{1}{k+1} \sum_{\gamma=r}^{r+k} s_{\gamma}$.

A bounded sequence $\left\{s_{k}\right\}$ is called almost Riesz summable to ' $s$ ' if the Riesz-transform of $\left\{s_{k}\right\}$ is almost convergent to a finite number ' $s$ ' (see King (1966) [3]).

The necessary and sufficient condition for the regularity of Riesz transform $\left(R, p_{n}\right)$ is that $P_{n} \rightarrow \infty$ as $n \rightarrow \infty$ ([27]; see Theorem 1.4.4 of Petersen (1966), p.10).

We write throughout the paper

$$
\begin{aligned}
& \psi_{x}(t)=f(x+t)-f(x-t)-2 f(x), \\
& p(y)=p_{[y]} \quad \text { and } \quad P(y)=P_{[y]}, \quad P_{\tau}=P(1 / t) .
\end{aligned}
$$

The notation $\tau=[1 / t]$ means the greatest integer contained in ' $1 / t$ '.

\section{Main result}

Hardy and Littlewood [28] have determined without verification that the class of functions $\operatorname{Lip}(\alpha, p)$ is the same as the class of functions approximable in the $L_{r}$-norm with degree of approximation $\mathrm{O}\left(n^{-\alpha}\right)$, by trigonometric polynomials of order $n$. A trigonometric Fourier approximation, began from a well-known theorem of approximation theory i.e. the Weierstrass approximation theorem, which has turned into an energizing interdisciplinary field of study throughout the previous 130 years. These approximations have expected vital new dimensions due to their extensive variety of uses in signal theory [29], in general and digital signal processing [30]; in particular, in view of classical Shannon sampling theorem [19]. The above mentioned examination inspired us to study further the problem of the approximation of functions in weighted Lipschitz class i.e. $W\left(L^{r}, \xi(t)\right)$. A few more applications of sharper estimates and trigonometric Fourier approximation can be found in $[19,29-33]$, and the references given therein. In this study, the summability transforms play a vital part, especially when the Fourier series of a signal (function) is non-convergent. During the past few decades, different strategies for planning digital filters have been suggested. It is worth to specify that the best-known techniques use as approximation criterion the minimization of the $L_{p}$-measure $1 \leq p \leq \infty$. Thus the designing of digital filters has been recognized as an approximation problem. Here we are interested in approximations of signals in $L_{1}$-space. In this paper, we utilize the idea of an almost Riesz summability transform of the Fourier series with a suitable set of conditions and prove the following theorem. 
Theorem 1 Assume $f$ is a $2 \pi$-periodic signal (function) and integrable in the sense of Lebesgue over $[0,2 \pi]$. Then the degree of approximation of $f \in W\left(L^{r}, \xi(t)\right)(r \geq 1)$-class with $0 \leq \beta \leq 1-1 / r$ by an almost Riesz means of its Fourier series (2) is given by

$$
\left\|\tau_{n, r}(f(t) ; x)-f(t)\right\|_{r}=\mathrm{O}\left(P_{n}^{\beta+1 / r} \xi\left(P_{n}^{-1}\right)\right) \quad \forall n>0,
$$

provided that the positive increasing function $\xi(t)$ has the following features:

$$
\{\xi(t) / t\} \text { is non-increasing in ' } t \text { ', }
$$

$$
\left(\int_{0}^{\pi / P_{n}}\left(\frac{\left|\psi_{x}(t)\right|}{\xi(t)}\right)^{r} \sin ^{\beta r}(t / 2) d t\right)^{1 / r}=\mathrm{O}(1)
$$

and

$$
\left(\int_{\pi / P_{n}}^{\pi}\left(\frac{t^{-\delta}\left|\psi_{x}(t)\right|}{\xi(t)}\right)^{r} d t\right)^{1 / r}=\mathrm{O}\left(P_{n}^{\delta}\right)
$$

where $\delta$ is an arbitrary number such that $(\beta-\delta) s-1>0, r^{-1}+s^{-1}=1,1 \leq r \leq \infty$, and conditions (5) and (6) hold uniformly in $x$.

Note 1 Using condition (4), we get the inequality $\xi\left(\frac{\pi}{P_{n}}\right) \leq \pi \xi\left(\frac{1}{P_{n}}\right)$, for $\left(\frac{\pi}{P_{n}}\right) \geq\left(\frac{1}{P_{n}}\right)$.

\section{Proof of Theorem 1}

The partial sum $s_{k, r}(f(t), x)$ of the Fourier series (2) is given by

$$
s_{k, r}(f(t), x)-f(t)=\frac{1}{2 \pi(k+1)} \int_{0}^{\pi} \psi(t) \frac{\cos r t-\cos (k+r+1) t}{2 \sin ^{2} t / 2} d t .
$$

Representing the almost Riesz means of partial sums $s_{k, r}(f(t), x)$ by $\tau_{n, r}(f(t) ; x)$, we can write

$$
\begin{aligned}
& \tau_{n, r}(f(t) ; x)-f(t) \\
& \quad=\frac{1}{P_{n}} \sum_{k=0}^{n} p_{k}\left\{s_{k, r}(f(t) ; x)-f(t)\right\} \\
& \quad=\frac{1}{2 \pi P_{n}} \int_{0}^{\pi} \psi(t) \sum_{k=0}^{n} \frac{p_{k}}{(k+1)} \frac{[\cos r t-\cos (k+r+1) t]}{2 \sin ^{2} t / 2} d t \\
& \quad=\frac{1}{2 \pi P_{n}}\left[\int_{0}^{\pi / P_{n}}+\int_{\pi / P_{n}}^{\pi}\right] \psi(t)\left(\sum_{k=0}^{n} \frac{p_{k}}{(k+1)} \frac{\sin (k+2 r+1) \frac{t}{2} \sin (k+1) \frac{t}{2}}{\sin ^{2} t / 2}\right) d t \\
& \quad=I_{1}+I_{2} \quad \text { (say). }
\end{aligned}
$$

Obviously

$$
\left|\psi_{x}(x+t)-\psi_{x}(t)\right| \leq|f(u+x+t)-f(u+x)|+|f(u-x-t)-f(u-x)| .
$$


Hence, by Minkowski's inequality, we get

$$
\begin{aligned}
& \left\{\int_{0}^{2 \pi}\left|\left(\psi_{x}(x+t)-\psi_{x}(t)\right) \sin ^{\beta}(x / 2)\right|^{r} d x\right\}^{1 / r} \\
& \leq\left\{\int_{0}^{2 \pi}\left|(f(u+x+t)-f(u+x)) \sin ^{\beta}(x / 2)\right|^{r} d x\right\}^{1 / r} \\
& \quad+\left\{\int_{0}^{2 \pi}\left|(f(u-x-t)-f(u-x)) \sin ^{\beta}(x / 2)\right|^{r} d x\right\}^{1 / r}=\mathrm{O}(\xi(t)) .
\end{aligned}
$$

Then $f \in W\left(L^{r}, \xi(t)\right) \Rightarrow \psi_{x} \in W\left(L^{r}, \xi(t)\right)$.

Here

$$
I_{1}=\frac{1}{2 \pi P_{n}} \int_{0}^{\pi / P_{n}} \psi_{x}(t) \sum_{k=0}^{n} \frac{p_{k}}{(k+1)} \frac{\sin (k+2 r+1) \frac{t}{2} \sin (k+1) \frac{t}{2}}{\sin ^{2} t / 2} d t .
$$

Using Hölder's inequality, $\psi_{x}(t) \in W\left(L^{r}, \xi(t)\right)$ over $[0, \pi]$, equation (5), Note 1, $(\sin t / 2)^{-1} \leq \pi / t$, for $0<t \leq \pi,|\sin n t| \leq n|\sin t| \forall t \in R, n \in N, r^{-1}+s^{-1}=1$ such that $1 \leq r \leq \infty$, we have

$$
\begin{aligned}
\left|I_{1}\right| \leq & \frac{1}{2 \pi P_{n}}\left\{\left(\int_{0}^{\pi / P_{n}}\left(\frac{\left|\psi_{x}(t)\right| \sin ^{\beta}(t / 2)}{\xi(t)}\right)^{r} d t\right)^{1 / r}\right\} \\
& \cdot\left\{\left(\lim _{\epsilon \rightarrow 0} \int_{\epsilon}^{\pi / P_{n}}\left(\frac{\xi(t)}{\sin ^{\beta}(t / 2)}\left|\sum_{k=0}^{n} \frac{p_{k}}{(k+1)} \frac{\sin (k+2 r+1) \frac{t}{2} \sin (k+1) \frac{t}{2}}{\sin ^{2} \frac{t}{2}}\right|\right)^{s} d t\right)\right\}^{1 / s} \\
= & \mathrm{O}(1)\left\{\lim _{\epsilon \rightarrow 0}\left(\int_{\epsilon}^{\pi / P_{n}}\left(\frac{\xi(t)}{t^{\beta+1}}\right)^{s} d t\right)\right\}^{1 / s} \\
= & \mathrm{O}\left(\xi\left(\frac{\pi}{P_{n}}\right)\right)\left\{\lim _{\epsilon \rightarrow 0} \int_{\epsilon}^{\pi / P_{n}} t^{-(1+\beta) s} d t\right\}^{1 / s} \\
= & \mathrm{O}\left(P_{n}^{\beta+1 / r} \xi\left(P_{n}^{-1}\right)\right) .
\end{aligned}
$$

Now

$$
\begin{aligned}
\left|I_{2}\right|= & \left(\frac{1}{2 \pi P_{n}}\right)\left\{\int_{\pi / P_{n}}^{\pi} \frac{t^{-\delta}\left|\psi_{x}(t)\right| \sin ^{\beta}(t / 2)}{\xi(t)}\right. \\
& \left.\cdot \frac{\xi(t)}{t^{-\delta} \sin ^{\beta}(t / 2)}\left|\sum_{k=0}^{n} \frac{p_{k}}{(k+1)} \frac{\sin (k+2 r+1) \frac{t}{2} \sin (k+1) \frac{t}{2}}{\sin ^{2}(t / 2)}\right|\right\} .
\end{aligned}
$$

Now again by Hölder's inequality, we find

$$
\begin{aligned}
\left|I_{2}\right| \leq & \frac{1}{2 \pi P_{n}}\left\{\int_{\pi / P_{n}}^{\pi}\left(\frac{t^{-\delta}\left|\psi_{x}(t)\right| \sin ^{\beta}(t / 2)}{\xi(t)}\right)^{r} d t\right\}^{1 / r} \\
& \cdot\left\{\int_{\pi / P_{n}}^{\pi}\left(\frac{\xi(t)}{t^{-\delta} \sin ^{\beta}(t / 2)}\left|\sum_{k=0}^{n} \frac{p_{k}}{(k+1)} \frac{\sin (k+2 r+1) \frac{t}{2} \sin (k+1) \frac{t}{2}}{\sin ^{2}(t / 2)}\right|\right)^{s} d t\right\}^{1 / s}
\end{aligned}
$$


and using $(\sin t / 2)^{-1} \leq \pi / t$, on $0<t \leq \pi,|\sin t / 2| \leq 1$, conditions (4), (6), Note 1 , and the second mean value theorem for integrals, we get

$$
\begin{aligned}
\left|I_{2}\right| & \leq \frac{1}{2 \pi P_{n}}\left\{\int_{\pi / P_{n}}^{\pi}\left(\frac{t^{-\delta}\left|\psi_{x}(t)\right|}{\xi(t)}\right)^{r} d t\right\}^{1 / r} \cdot\left\{\int_{\pi / P_{n}}^{\pi}\left(\frac{\xi(t)}{t^{-\delta+\beta+1}} P_{n}\right)^{s} d t\right\}^{1 / s} \\
& =\mathrm{O}\left(P_{n}^{\delta}\right)\left\{\int_{\pi / P_{n}}^{\pi}\left(\frac{\xi(t)}{t^{-\delta+\beta+1}}\right)^{s} d t\right\}^{1 / s} \\
& =\mathrm{O}\left(P_{n}^{\delta}\right)\left\{\int_{1 / \pi}^{P_{n} / \pi}\left(\frac{\xi(1 / y)}{y^{\delta-\beta-1}}\right)^{s} \frac{d y}{y^{2}}\right\}^{1 / s}, \quad \text { putting } t=1 / y \\
& =\mathrm{O}\left\{\frac{P_{n}^{\delta+1}}{\pi} \xi\left(\frac{\pi}{P_{n}}\right)\right\}\left\{\int_{\epsilon_{1}}^{P_{n} / \pi} y^{(\beta-\delta) s-2} d y\right\}^{1 / s} \text { for some } \frac{1}{\pi}<\epsilon_{1}<\frac{P_{n}}{\pi} \\
& =\mathrm{O}\left\{\frac{P_{n}^{\delta+1}}{\pi} \xi\left(P_{n}^{-1}\right)\right\}\left\{\frac{\left(P_{n}\right)^{(\beta-\delta) s-1}-\left(\epsilon_{1}\right)^{(\beta-\delta) s-1}}{(\beta-\delta) s-1}\right\}^{1 / s} \\
& =\mathrm{O}\left\{P_{n}^{\delta+1} \xi\left(P_{n}^{-1}\right) \cdot P_{n}^{\beta-\delta-1 / s}\right\}=\mathrm{O}\left\{P_{n}^{\beta+1 / r} \xi\left(P_{n}^{-1}\right)\right\} .
\end{aligned}
$$

On collecting (7)-(9), we have

$$
\left|\tau_{n, r}(f(t) ; x)-f(t)\right|=\mathrm{O}\left\{P_{n}^{\beta+1 / r} \xi\left(P_{n}^{-1}\right)\right\}
$$

Now, using the $L_{r}$-norm of function, we obtain

$$
\begin{aligned}
\left\|\tau_{n, r}(f(t) ; x)-f(t)\right\|_{r} & =\left\{\int_{0}^{2 \pi}\left|\tau_{n, r}(f(t) ; x)-f(t)\right|^{r} d x\right\}^{1 / r} \\
& =\mathrm{O}\left\{\int_{0}^{2 \pi} P_{n}^{\beta+1 / r} \xi\left(P_{n}^{-1}\right) d x\right\}^{1 / r} \\
& =\mathrm{O}\left\{P_{n}^{\beta+1 / r} \xi\left(P_{n}^{-1}\right)\right\}\left(\int_{0}^{2 \pi} d x\right)^{1 / r} \\
& =\mathrm{O}\left\{P_{n}^{\beta+1 / r} \xi\left(P_{n}^{-1}\right)\right\} .
\end{aligned}
$$

This completes the proof of Theorem 1 .

\section{Corollaries}

The following corollaries can be derived from our main Theorem 1.

Corollary 1 If $\beta=0$, then the generalized weighted Lipschitz $W\left(L^{r}, \xi(t)\right)(r \geq 1)$-class reduces to the class $\operatorname{Lip}(\xi(t), r)$ and the error in approximation of a function $f$, belonging to the class $\operatorname{Lip}(\xi(t), r)$, is given by

$$
\left\|\tau_{n, r}(f(t) ; x)-f(t)\right\|_{r}=\mathrm{O}\left(P_{n}^{1 / r} \xi\left(P_{n}^{-1}\right)\right) .
$$

Proof The result follows by putting $\beta=0$ in (3); we get

$$
\left\{\int_{0}^{2 \pi}\left|\tau_{n, r}(f(t) ; x)-f(t)\right|^{r} d x\right\}^{1 / r}=\mathrm{O}\left(P_{n}^{1 / r} \xi\left(P_{n}^{-1}\right)\right), \quad r \geq 1
$$


Thus, we get

$$
\left|\tau_{n, r}(f(t) ; x)-f(t)\right| \leq\left\{\int_{0}^{2 \pi}\left|\tau_{n, r}(f(t) ; x)-f(t)\right|^{r} d x\right\}^{1 / r}=\mathrm{O}\left(P_{n}^{1 / r} \xi\left(P_{n}^{-1}\right)\right), \quad r \geq 1
$$

This completes the proof of Corollary 1.

Corollary 2 If $\beta=0, \xi(t)=t^{\alpha}, 0<\alpha \leq 1$, then the generalized weighted Lipschitz $W\left(L^{r}, \xi(t)\right)(r \geq 1)$-class reduces to the class $\operatorname{Lip}(\alpha, r),(1 / r)<\alpha<1$ and the degree of approximation of a function $f$ belonging to the class $\operatorname{Lip}(\alpha, r)$, is given by

$$
\left|\tau_{n, r}(f(t) ; x)-f(t)\right|=\mathrm{O}\left(P_{n}^{-\alpha+1 / r}\right) .
$$

Proof Putting $\beta=0, \xi(t)=t^{\alpha}, 0<\alpha \leq 1$ in Theorem 1 , we have

$$
\left\|\tau_{n, r}(f(t) ; x)-f(t)\right\|_{r}=\left\{\int_{0}^{2 \pi}\left|\tau_{n, r}(f(t) ; x)-f(t)\right|^{r} d x\right\}^{1 / r}
$$

or

$$
\mathrm{O}\left(P_{n}^{\beta+1 / r} \xi\left(P_{n}^{-1}\right)\right)=\left\{\int_{0}^{2 \pi}\left|\tau_{n, r}(f(t) ; x)-f(t)\right|^{r} d x\right\}^{1 / r}
$$

or

$$
\mathrm{O}(1)=\left\{\int_{0}^{2 \pi}\left|\tau_{n, r}(f(t) ; x)-f(t)\right|^{r} d x\right\}^{1 / r} \mathrm{O}\left(\frac{1}{P_{n}^{\beta+1 / r} \xi\left(P_{n}^{-1}\right)}\right)
$$

since otherwise the right-hand side of the above equation will not be $\mathrm{O}(1)$.

Hence

$$
\left|\tau_{n, r}(f(t) ; x)-f(t)\right|=\mathrm{O}\left(P_{n}^{1 / r} P_{n}^{-\alpha}\right)=\mathrm{O}\left(P_{n}^{-\alpha+1 / r}\right)
$$

which is one of the results of Mishra et al. [34].

Corollary 3 If $\beta=0, \xi(t)=t^{\alpha}$ for $0<\alpha<1$ and $r \rightarrow \infty$ in (3), then $f \in \operatorname{Lip} \alpha$. In this case, the degree of approximation of a function $f$ belonging to the class $\operatorname{Lip} \alpha(0<\alpha<1)$ is given by

$$
\left|\tau_{n, r}(f(t) ; x)-f(t)\right|=\mathrm{O}\left(P_{n}^{-\alpha}\right)
$$

Proof For $r \rightarrow \infty$ in Corollary 2, we get

$$
\left\|\tau_{n, r}(f(t) ; x)-f(t)\right\|_{\infty}=\sup _{0 \leq x \leq 2 \pi}\left|\tau_{n, r}(f(t) ; x)-f(t)\right|=\mathrm{O}\left(P_{n}^{-\alpha}\right) .
$$

Thus, we have

$$
\begin{aligned}
\left|\tau_{n, r}(f(t) ; x)-f(t)\right| & \leq\left\|\tau_{n, r}(f(t) ; x)-f(t)\right\|_{\infty} \\
& =\sup _{0 \leq x \leq 2 \pi}\left|\tau_{n, r}(f(t) ; x)-f(t)\right|=\mathrm{O}\left(P_{n}^{-\alpha}\right) .
\end{aligned}
$$


This completes the proof of Corollary 3.

\section{Conclusion}

A few interesting results with respect to the error in the approximation of periodic signals (functions) belonging to the different Lipschitz classes by almost convergent sequences have been audited. Further, a suitable arrangement of limitations has been examined to the errors in this direction. Some interesting uses of the approximation of signals utilized as a part of this manuscript have additionally been highlighted. The consequences of our theorem and corollaries are broader as opposed to the consequences of whatever other already demonstrated lemmas and theorems; they will be an advance of the theory of applications of summability analysis in the theory of approximations. The study of the degree of approximation of signals in other function spaces such as the Hölder, Zygmund classes and so on, might be of future interest for investigators in this direction. The scientists and experts working or expecting to work in the areas of analysis and its applications will find this exploration article to be altogether important. Henceforth, the results so settled may be found to be helpful and fascinating under circumstances showing up in the literature on mathematics and applications.

\section{Competing interests}

The authors declare that they have no competing interests regarding the publication of this manuscript.

\section{Authors' contributions}

$\mathrm{D}$ and L-IP computed the approximation properties of the periodic functions by an almost Riesz means of the Fourier series in the Lipschitz classes. Both authors contributed equally and significantly in writing this manuscript. Both authors read and approved the final manuscript.

\section{Author details}

'SQC and OR Unit, Indian Statistical Institute, 203 B.T. Road, Kolkata-700 108, West Bengal, India. ${ }^{2}$ Department of Mathematics and Computer Science, North University Center of Baia Mare, Technical University of Cluj Napoca, Victoriei 76, Baia Mare, 430122, Romania.

\section{Acknowledgements}

The first author is thankful to have been enabled to carry out this work under the project on Optimization and Reliability Modelling of Indian Statistical Institute.

Received: 12 February 2016 Accepted: 5 June 2016 Published online: 21 June 2016

\section{References}

1. Lorentz, GG: A contribution to the theory of divergent series. Acta Math. 80, 167-190 (1948)

2. Ahmad, ZU, Mursaleen, M: An application of Banach limits. Proc. Am. Math. Soc. 103(1), 244-246 (1988)

3. King, JP: Almost summable sequences. Proc. Am. Math. Soc. 17, 1219-1225 (1966)

4. Mohapatra, RN: Quantitative results on almost convergence of a sequence of positive linear operators. J. Approx. Theory 20(3), 239-250 (1977)

5. Mohapatra, RN: Functions of class Lip $(\alpha, p)$ and their Taylor mean. J. Approx. Theory 45(4), 363-374 (1985)

6. Nanda, S: Some sequence spaces and almost convergence. J. Aust. Math. Soc. A 22, 446-455 (1976)

7. Mursaleen, M, Mohiuddine, SA: Convergence Methods for Double Sequences and Applications. Springer, Berlin (2014)

8. Mohiuddine, SA: An application of almost convergence in approximation theorems. Appl. Math. Lett. 24, 1856-1860 (2011)

9. Edely, OHH, Mohiuddine, SA, Noman, AK: Korovkin type approximation theorems obtained through generalized statistical convergence. Appl. Math. Lett. 23, 1382-1387 (2010)

10. Mohiuddine, SA, Alotaibi, A: Korovkin second theorem via statistical summability $(C ; 1)$. J. Inequal. Appl. 2013, Article ID 149 (2013)

11. Das, G, Kuttner, B, Nanda, S: Some sequence spaces and absolute almost convergence. Trans. Am. Math. Soc. 283(2), 729-739 (1984)

12. Maddox, IJ: A new type of convergence. Math. Proc. Camb. Philos. Soc. 83, 61-64 (1978)

13. Maddox, IJ: Elements of Functional Analysis. Cambridge University Press, Cambridge (1970)

14. Schaefer, P: Almost convergent and almost summable sequences. Proc. Am. Math. Soc. 20, $51-54$ (1969)

15. Khan, $\mathrm{HH}$ : On the degree of approximation to a function belonging to weighted $\left(L^{p}, \xi(t)\right)$ class. Aligarh Bull. Math. 3/4, 83-88 (1973-1974)

16. Khan, $\mathrm{HH}$ : On the degree of approximation to a function by triangular matrix of its conjugate Fourier series II. Indian J. Pure Appl. Math. 6, 1473-1478 (1975) 
17. Mishra, VN, Mishra, LN: Trigonometric approximation of signals (functions) in $L_{p}(p \geq 1)$-norm. Int. J. Contemp. Math. Sci. 7(19), 909-918 (2012)

18. Mishra, VN, Khatri, K, Mishra, LN, Deepmala: Trigonometric approximation of periodic signals belonging to generalized weighted Lipschitz $W^{\prime}\left(L^{r}, \xi(t)\right)(r \geq 1)$-class by Nörlund-Euler $\left(N, p_{n}\right)(E, q)$ operator of conjugate series of its Fourier series. J. Class. Anal. 5(2), 91-105 (2014)

19. Mishra, LN, Mishra, VN, Khatri, K, Deepmala: On the trigonometric approximation of signals belonging to generalized weighted Lipschitz $W\left(L^{r}, \xi(t)\right)(r \geq 1)$-class by matrix $\left(C^{1} \cdot N_{p}\right)$ operator of conjugate series of its Fourier series. Appl. Math. Comput. 237, 252-263 (2014)

20. Mishra, VN, Khan, HH, Khatri, K, Mishra, LN: Degree of approximation of conjugate of signals (functions) belonging to the generalized weighted Lipschitz $W\left(L_{r}, \xi(t)\right)(r \geq 1)$-class by $(C, 1)(E, q)$ means of conjugate trigonometric Fourier series. Bull. Math. Anal. Appl. 5(4), 40-53 (2013)

21. Mishra, VN, Khan, HH, Khatri, K, Mishra, LN: On approximation of conjugate of signals (functions) belonging to the generalized weighted $W\left(L_{r}, \xi(t)\right)(r \geq 1)$-class by product summability means of conjugate series of Fourier series. Int. J. Math. Anal. 6(35), 1703-1715 (2012)

22. Mishra, VN, Khatri, K, Mishra, LN: Approximation of functions belonging to the generalized Lipschitz class by $C^{1} \cdot N_{p}$ summability method of conjugate series of Fourier series. Mat. Vesn. 66(2), 155-164 (2014)

23. Deepmala, Mishra, LN, Mishra, VN: Trigonometric approximation of signals (functions) belonging to the $W\left(L_{r}, \xi(t)\right)(r \geq 1)$-class by $(E, q)(q>0)$-means of the conjugate series of its Fourier series. Glob. J. Math. Sci. 2(2), 61-69 (2014)

24. MCFadden, L: Absolute Nörlund summability. Duke Math. J. 9, 168-207 (1942)

25. Zygmund, A: Trigonometric Series, vols. I and II, 2nd edn. Cambridge University Press, London (1968)

26. Sharma, PL, Qureshi, K: On the degree of approximation of a periodic function by almost Riesz means. Ranchi Univ. Math. J. 11, 29-43 (1980)

27. Petersen, GM: Regular Matrix Transformations. McGraw-Hill, London (1966)

28. Hardy, GH, Littlewood, JE: A convergence criterion for Fourier series. Math. Z. 28, $612-634$ (1928)

29. Proakis, JG: Digital Communications. McGraw-Hill, New York (1995)

30. Psarakis, EZ, Moustakides, GV: An $L_{2}$-based method for the design of 1-D zero phase FIR digital filters. IEEE Trans. Circuits Syst. I, Fundam. Theory Appl. 44(7), 591-601 (1997)

31. Chen, WK: Mathematics for Circuits and Filters. CRC Press, Boca Raton (2000)

32. Banach, S: Théorie des Operations Lineaires. Instytut Matematyczny Polskiej Akademi Nauk, Warsaw (1932)

33. Gil', Ml: Estimates for entries of matrix valued functions of infinite matrices. Math. Phys. Anal. Geom. 11, 175-186 (2008)

34. Mishra, VN, Khan, $\mathrm{HH}$, Khan, IA, Mishra, LN: On the degree of approximation of signals of $\operatorname{Lip}(\alpha, r)(r \geq 1)$-class by almost Riesz means of its Fourier series. J. Class. Anal. 4(1), 79-87 (2014)

\section{Submit your manuscript to a SpringerOpen ${ }^{\circ}$ journal and benefit from:}

- Convenient online submission

Rigorous peer review

- Immediate publication on acceptance

- Open access: articles freely available online

- High visibility within the field

- Retaining the copyright to your article 